\title{
In Vivo Assessment of Acute Microvascular Injury after Reperfusion of Ischemic Tibialis Anterior Muscle of the Hamster
}

\author{
Louis M. MESSINA, M.D. \\ With the technical assistance of Ane Gardner, B.S. \\ Department of Surgery, the Section of Vascular Surgery, and the Conrad Jobst Vascular Research Laboratories, \\ University of Michigan Medical School, Ann Arbor, Michigan 48109-0329
}

Presented at the Annual Meeting of the Association for Academic Surgery, Louisville, Kentucky, November $15-18,1989$

The purposes of this study were to develop an in vivo model of skeletal muscle ischemia-reperfusion to assess the patterns of microvascular injury, to evaluate a scoring system that permits quantitation of this injury, and to determine in vivo the extent of white blood cell adhesion within the microcirculation during the acute postreperfusion period. Syrian golden hamsters underwent 3.0 or $4.5 \mathrm{hr}$ of lower extremity ischemia without anticoagulation. The microcirculation of the tibialis anterior muscle was visualized by fluorescent intravital microscopy $(700 X)$. During the first $1.5 \mathrm{hr}$ of reperfusion the microvascular injury was scored by a grading system based upon the extent of extravasation of fluorosceinlabeled albumin and the degree and level of microvessel obstruction. To correlate the observed changes in the microcirculation to changes in the whole muscle, in a separate group of animals, pH changes in the tibialis anterior muscle were measured at the same time intervals under identical experimental conditions as the microvascular measurements. White blood cells were transiently fluoresced at $1.5 \mathrm{hr}$ after reperfusion by intravenous acridine red and the number of white blood cells rolling (rollers) or sticking (stickers) to the endothelium during a 30-sec observation period was recorded. Two distinct patterns of microvascular injury were seen: after $3.0 \mathrm{hr}$ of ischemia there was a progressive extravasation, some capillary but no arteriolar or venular obstruction, flow velocities increased over time; after $4.5 \mathrm{hr}$ of ischemia there was a greater heterogeneity of injury, primary "no reflow," extensive capillary, arteriolar, and venular obstruction, as well as a progressive decline in flow velocities. Thrombosis of microvessels was rare. There was no inflow vessel thrombosis. The scoring system showed significant differences, mean (SE), in both the extent of early (30 $\mathrm{min})$ and late $(90 \mathrm{~min})$ injuries for the $3.0-\mathrm{hr}$ (early $1.4(0.6)$ vs late $2.0(0.5)$ ) and the 4.5 -hr periods of ischemia (early $2.3(0.9)$ vs late $3.0(0.8)$ ). In addition, pH measurements of the tibialis anterior paralleled the changes observed in the microcirculation. There was a significant increase in the number of rolling WBCs, 14.3 (10.3), in the animals undergoing $3.0 \mathrm{hr}$ of ischemia, and in both the number of rollers, $7.4(4.3)$, and stickers, 4.0 (1.9), in the animals undergoing $4.5 \mathrm{hr}$ of ischemia compared to those of controls, $1.7(2.4)$ and $1.9(1.5)$. These results are the first in vivo observations of the pattern of microvascular injury and the extent of white blood cell adhesion in a true muscle of locomotion. We believe that this model will allow further clarification of the mechanisms responsible for the microvascular injury that occurs after reperfusion of ischemic skeletal muscle. c) 1980 Academic Press, Inc.

\section{INTRODUCTION}

Ischemia is an important cause of skeletal muscle injury and resultant patient disability. Much of the experimental work on ischemia-reperfusion injury has focused on changes in the parenchymal cell either at the organ or at the cellular level and, more recently, on the role of white blood cells in the amplification of ischemia-reperfusion injury [1-5]. Little is known about the changes in the microcirculation during reperfusion of ischemic tissue. Microvascular injury after ischemia-reperfusion remains poorly understood in part because of a lack of a satisfactory in vivo, clinically relevant experimental model. The purposes of this study were to develop an in vivo model of skeletal muscle ischemia-reperfusion injury to assess the patterns of microvascular injury, to evaluate a scoring system that permits quantitation of this injury, and to determine in vivo the extent of white blood cell adhesion in the acute postreperfusion period. To correlate the observed changes in the microcirculation that occur after reperfusion to changes in the whole muscle, $\mathrm{pH}$ measurements of the tibialis anterior were made under identical experimental conditions in a separate group of animals.

\section{METHODS}

Syrian golden hamsters $(90-100 \mathrm{~g})$ underwent a modified form of tourniquet ischemia of the lower extremity. 
After the induction of anesthesia by the intraperitoneal injection of pentobarbitol $(0.1 \mathrm{mg} / \mathrm{g} / \mathrm{body}$ wt $)$ the femoral artery was occluded with a microvascular clamp. The femoral nerve and vein remained free. Bands were placed around the thigh musculature at the level of the inguinal ligament to obliterate collateral flow. No heparin anticoagulation was used.

After the initial surgical preparation the hamster was transferred to the stage of a Wayland-Frasher intravital microscope (Fig. 1). The animals were ventilated mechanically through a tracheostomy tube at a rate and volume sufficient to maintain arterial blood gases within normal physiologic limits. Arterial blood was obtained for $\mathrm{pH}$ and gas analysis from a catheter placed into the cervical carotid artery. Continuous anesthesia was maintained by constant infusion of sodium pentobarbitol ( 9 $\mathrm{mg} / \mathrm{ml}$ ) at a rate of $0.0073-0.013 \mathrm{ml} / \mathrm{min}$ through a catheter in the jugular vein. A rectal temperature probe was placed and connected to a servo-controlled heating pad which maintained rectal temperature at $37^{\circ} \mathrm{C}$. Either lower leg was then fixed to a plexiglass platform in a horizontal plane (Fig. 1). The skin and then the tendinous portion of the biceps femoris muscle were incised directly over the tibialis anterior. This muscle was continuously bathed in a bicarbonate-buffered salt solution from a reservoir maintained at $35^{\circ} \mathrm{C}$ and equilibrated with $5 \% \mathrm{CO}_{2}$ and $95 \%$ nitrogen. This suffusate had a millimolar composition of $\mathrm{NaCl} 130.9, \mathrm{KCl} 4.7, \mathrm{CaCl}_{2} 2.0, \mathrm{MgSO}_{4} 1.2$, and $\mathrm{NaHCO}_{3} 20.0$.

The microcirculation was visualized by the intravenous administration of fluorescein-labeled bovine serum albumin (10-15 mg/100 mg body wt). The muscle was epiilluminated with a 100-W mercury lamp (Leitz) connected to an American Optical Illuminator for incident light fluoresence (Model 2070). The microcirculation was viewed through a close-circuit video system consisting of a lowlight level Dage-Sit 66 camera (Dage-MTI, Inc., Michigan City, IN) connected to a four-head video recorder (Panasonic AG 1950) and a high resolution television monitor (Panasonic TR 124).

Acridine red (Chroma, Stuttgart, FRG) ( $5 \mu \mathrm{g} / 100 \mathrm{mg}$ body wt) was administered intravenously to transiently fluoresce white blood cells at a wavelength (excitation, $546 \mathrm{~nm}$; emission, $590 \mathrm{~nm}$ ) higher than that of fluorescein (excitation, $490 \mathrm{~nm}$; emission, $515 \mathrm{~nm}$ ). Although acridine red also fluoresces platelets, because of their size and velocity they require sophisticated techniques in order to be observed clearly [6].

In order to measure $\mathrm{pH}$ changes in the tibialis anterior separate groups of animals underwent similar durations of ischemia and reperfusion under identical experimental conditions but instead the $\mathrm{pH}$ of the tibialis anterior was monitored. A pH electrode (Vascular Tech., Inc., Chelmsford, MA) attached to an Omega pH meter (PHB .83) was inserted into the tibialis anterior prior to the onset of ischemia. Measurements were made at the onset

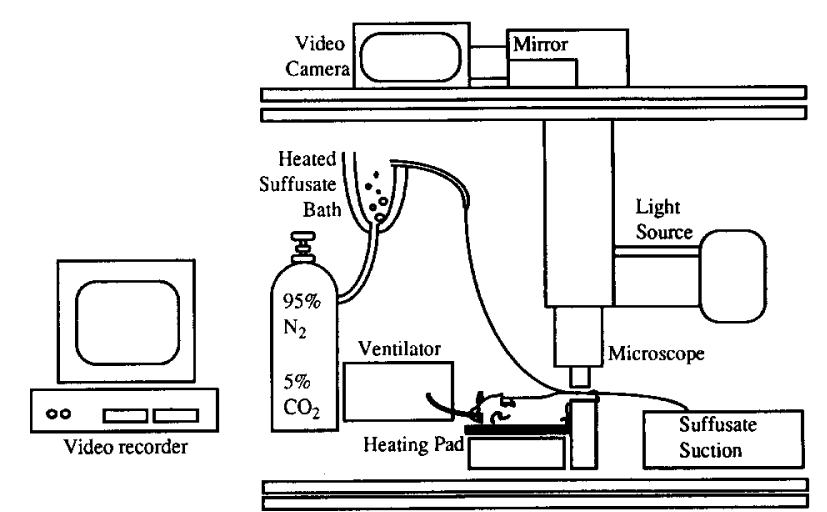

FIG. 1. Schematic illustration of the Wayland-Frasher intravital microscope and the experimental preparation.

of ischemia, at the time of reperfusion, and at 30 and 90 min after reperfusion.

\section{EXPERIMENTAL PROTOCOL}

Hamsters underwent either a $3.0-\mathrm{hr}$ or a 4.5 -hr period of ischemia. The microcirculation was visualized and studied during the initial $1.5 \mathrm{hr}$ after reperfusion. A separate group of hamsters served as sham controls in which the hamsters underwent an identical surgical preparation including complete dissection of the groin, placement of bands around the thigh musculature, and a $3.0-\mathrm{hr}$ control observation period. In all groups the exposed surface of the tibialis was videorecorded at $700 \mathrm{X}$ and a minimum of 12 randomly chosen fields were recorded at 30 and 90 min after reperfusion.

The degree of microvascular injury was scored according to the following system: Grade I ( $1 \mathrm{pt})$-mild to moderate extravasation of fluorescein-labeled albumin but full reflow, i.e., less than three capillaries obstructed per field; Grade II (2 pt) - extensive extravasation; Grade III (2.5 pt)-between three and five capillaries obstructed per field but the arterioles and venules remain patent; Grade IV $(3 \mathrm{pt})$ - extensive capillary obstruction, greater than five capillaries per field, some arteriolar and venular obstruction, extravasation may or may not be present; and Grade $\mathrm{V}(4 \mathrm{pt})$-no reflow, that is greater than $90 \%$ of the observed area was without any flow.

White blood cell adhesion to the microvascular endothelium was measured at $1.5 \mathrm{hr}$ after reperfusion. Fiftymicrometer segments of $20-\mu \mathrm{m}$ - to $30-\mu \mathrm{m}$-diameter venules were videorecorded for $30 \mathrm{sec}$ and the number of white blood cells rolling along the endothelium (rollers) or those adherent or sticking to (stickers) the endothelium per $30 \mathrm{sec}$ observation period was recorded [7]. Stickers indicate a greater degree of adhesion to the endothelium than those slowly rolling along the vessel wall versus those white cells flowing freely through the vessel.

Results will be reported as mean (standard error). Statistical analysis was performed using paired and un- 


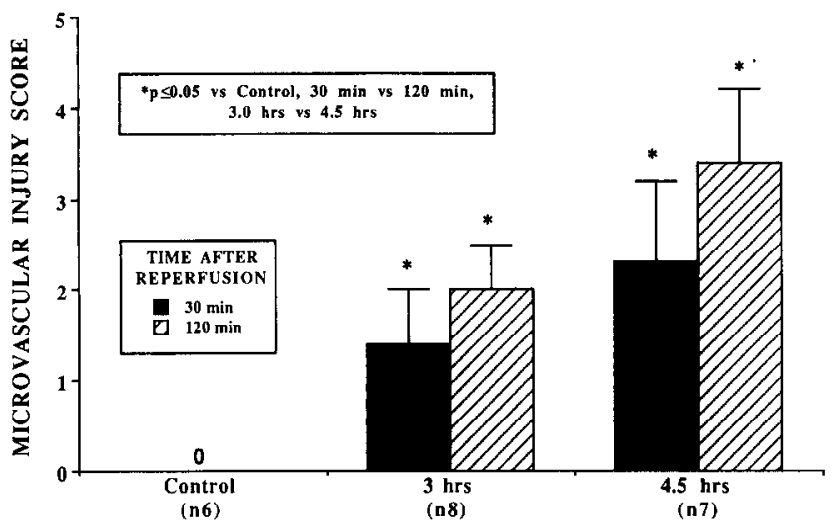

SCORE OF MICROVASCULAR INJURY AFTER REPERFUSION

FIG. 2. The scoring system showed a significant increase in the degree of microvascular injury for the $3.0-\mathrm{hr}$ and the $4.5-\mathrm{hr}$ periods of ischemia between the 30 - and the 90 -min observations during reperfusion. There was also a significant increase in the score of microvascular injury between the early and the late time periods of the $3.0-\mathrm{hr}$ and 4.5 $\mathrm{hr}$ groups. There was no microvascular injury in the sham control animals.

paired Student's $t$ test. $P$ values less than or equal to 0.05 were considered significant.

\section{RESULTS}

Two distinct patterns of microvascular injury were observed. After $3.0 \mathrm{hr}$ of ischemia, the reperfusion of the skeletal muscle microcirculation was characterized by progressive extravasation of fluoroscein-labeled albumin, some capillary but no arteriolar or venular obstruction; in six of the seven animals the blood flow velocity increased over the 2 -hr observation. Some capillaries remained obstructed for the entire period of observation. The dominant change in the microcirculation after $3 \mathrm{hr}$ of ischemia was extravasation. This microvascular injury appeared reversible. The pattern of injury after $4.5 \mathrm{hr}$ of ischemia appeared irreversible within the time frame of these experiments. It was characterized by a more heterogeneous pattern of injury. There was a lesser degree of extravasation because of a greater degree of progressive capillary obstruction, or primary no reflow; that is, no return of circulation to an area of the muscle upon release of the clamps. Under conditions of primary no reflow a whole microvascular unit consisting of the arteriole, capillaries, and venule was usually absent. For the $4.5-\mathrm{hr}$ period of ischemia the dominant change was loss of perfusion in the microcirculation. This loss of perfusion was due largely to loss of capillary flow. The loss of perfusion in the venules and arterioles usually came either as part of an area of primary no reflow or after near complete obstruction of all the capillaries for a particular microvascular unit. Thrombosis of microvessels was seen rarely. All animals in both groups were found to have patent inflow vessels at the conclusion of each experiment.
The scoring system provided a sensitive means by which to quantitate the extent of microvascular injury (Fig. 2). There was a significant increase in the degree of microvascular injury for both the $3.0-\mathrm{hr}$ and the $4.5-\mathrm{hr}$ periods of ischemia between the early $(30 \mathrm{~min}$ ) and the late (90 min) observations. In addition, there was a significant increase in the score of microvascular injury between the early and the late time periods of the $3.0-\mathrm{hr}$ and $4.5-\mathrm{hr}$ groups. No microvascular injury was seen in the sham control animals.

Acridine red transiently labeled the white blood cells and permitted assessment of their adhesion to microvessels (Fig. 3). There was a significant increase in the degree of white cell adhesion after both the $3.0-$ and the $4.5-\mathrm{hr}$ periods of ischemia (Table 1). Compared to sham controls there was a statistically significant increase in the number of rolling white cells in the group of animals which underwent $3 \mathrm{hr}$ of ischemia. In the group of animals that underwent $4.5 \mathrm{hr}$ of ischemia there was a significant increase in both the number of WBC rollers and WBC stickers compared to control measurements. Because of the relative reduction in the total flow to the muscle after $4.5 \mathrm{hr}$ ischemia, the total flux of WBCs through the microcirculation appeared to be reduced. We believe that this may have accounted for the fewer WBC rollers in the 4.5-hr group than in the 3.0-hr ischemia group.

The $\mathrm{pH}$ changes of the tibialis anterior that occurred during ischemia and reperfusion paralleled the changes that occurred in the microcirculation under identical experimental conditions (Fig. 4). The $\mathrm{pH}$ fell to a lower level, $6.3 \mathrm{pH}$ units, after $4.5 \mathrm{hr}$ of ischemia than after 3.0 $\mathrm{hr}$ of ischemia, $6.6 \mathrm{pH}$ units, although this did not reach statistical significance $(P=0.07)$. Importantly, after 90 min of reperfusion, there was a recovery in the $\mathrm{pH}$ to the baseline level in the group of animals that underwent 3 $\mathrm{hr}$ of ischemia. However, in the animals that underwent $4.5 \mathrm{hr}$ of ischemia the $\mathrm{pH}$ failed to recover to the baseline level, thus paralleling the microvascular changes in which the microvascular injury after $4.5 \mathrm{hr}$ of ischemia appeared irreversible. In three animals in which we continued observations beyond this time period, no further significant increase in the $\mathrm{pH}$ occurred.

TABLE 1

White Blood Cell Adhesion after Reperfusion

\begin{tabular}{lcc}
\hline $\begin{array}{c}\text { Duration of } \\
\text { ischemia }\end{array}$ & $\begin{array}{c}\text { Rollers } \\
\text { (no./50 } \mu \mathrm{m} / 30 \mathrm{in.})\end{array}$ & $\begin{array}{c}\text { Stickers } \\
\text { (no. } / 50 \mu \mathrm{m} / 30 \mathrm{in} .)\end{array}$ \\
\hline Control $(n=6)$ & $1.7(2.4)$ & $1.9(1.5)$ \\
$3 \mathrm{hr}(n=12)$ & $14.3(10.3)^{*}$ & $1.4(1.2)$ \\
$4.5 \mathrm{hr}(n-4)$ & $7.4(4.3)^{*}$ & $4.0(1.9)^{*}$ \\
\hline
\end{tabular}

* Unpaired $t$ test $P \leqslant 0.05$ vs control. 

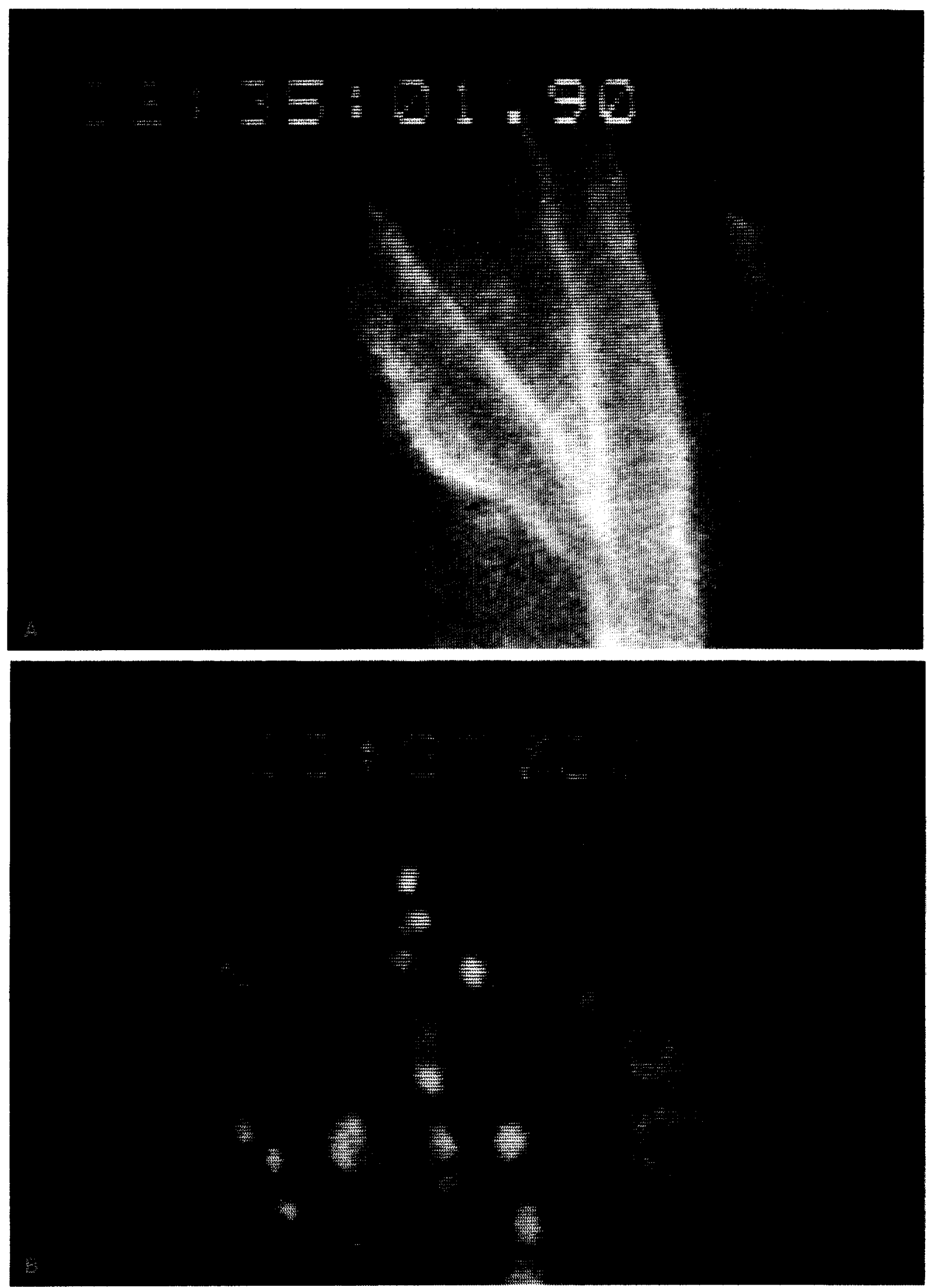

FIG. 3. (A) Photograph of videotape showing a draining venule using filters for fluorescein which shows the fluorescently labeled plasma albumin. (B) The same venule as it appears using the filters for acridine red. One can observe extensive adhesion of white cells to the endothelium. 


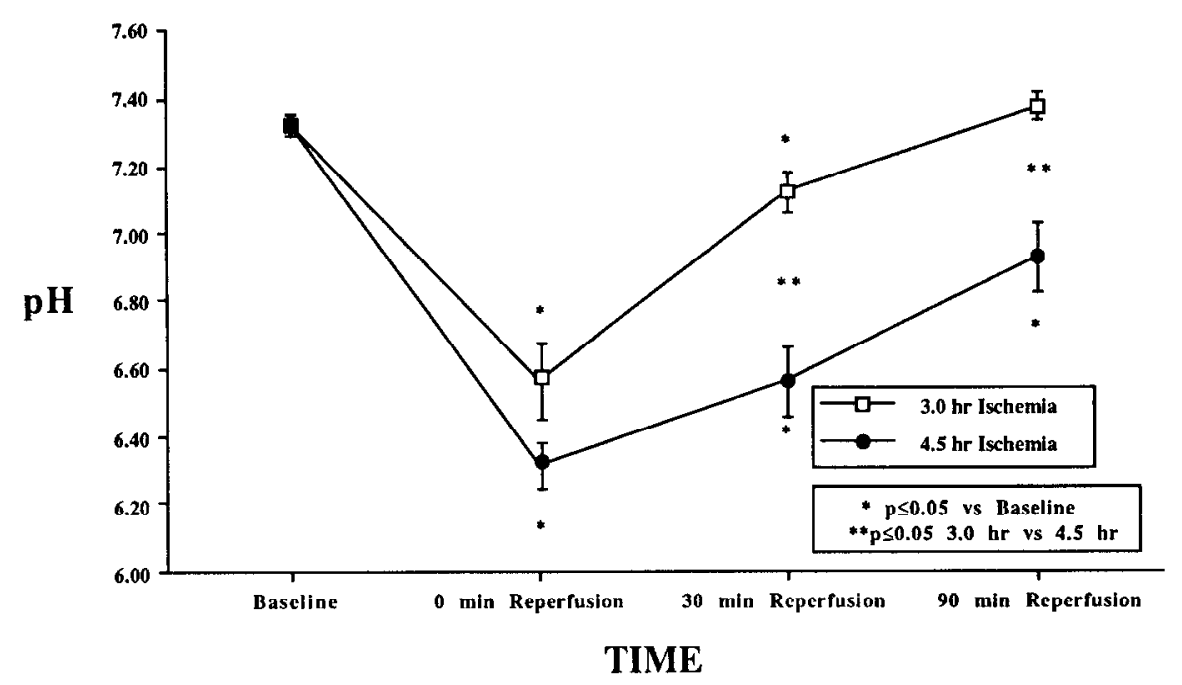

FIG. 4. This graph illustrates the changes in $\mathrm{pH}$ for the 3.0 -hr and 4.5 -hr periods of ischemia and subsequent reperfusion. The pH in the group of animals that underwent 4.5 -hr periods $(n=8)$ of ischemia failed to return to baseline values, whereas the $\mathrm{pH}$ did return to baseline values in the group of animals that underwent $3.0 \mathrm{hr}(n=5)$ of ischemia.

\section{DISCUSSION}

This experimental model permitted serial, in vivo measurements of microvascular injury after ischemia-reperfusion injury of the tibialis anterior of the hamster. In our model, ischemia was induced by a modified form of tourniquet ischemia. The femoral artery was occluded with a microvascular clamp. There was no venous or lymphatic obstruction, nor was there any nerve compression. Bands were placed only around the very proximal thigh musculature at a site remote from the tibialis anterior. Thus, the ischemia induced in the tibialis anterior parallels that which occurs clinically during acute ischemia of the lower extremity. Fluorescent intravital microscopy provided a means by which to observe in vivo the pattern of microvascular injury. The scoring system devised to grade the degree of microvascular injury, based upon the extent of extravasation as well as the level of microvessel obstruction, provided a sensitive means by which to differentiate different grades of microvascular injury (Fig. 2).

The mechanisms responsible for the microvascular injury after reperfusion of ischemic tissue have not been defined. In addition, there is no clear description of the changes in the microcirculation that occur after reperfusion of ischemic tissue. Recent work [8-10] has focused only on the loss of capillary flow. These studies have involved either postmortem analysis of histologic sections [10] or in vivo assessments of capillary flow [8,9]. We chose not to quantify only the capillary flow because we have found the changes in the microcirculation to be more complex than that. Although progressive capillary obstruction is a prominent event, there is also arteriolar and venular obstruction and areas which never have return of the microcirculation at all. Thus, an assessment of mi- crovascular perfusion during reperfusion of ischemic tissue must take into account both the level and the extent of microvessel obstruction. The scoring system employed in this study reflects these changes in the perfusion of the microcirculation as well as the extent of extravasation. In addition, as the pattern of injury in the microcirculation reflects the degree of ischemia, we studied time periods which embraced both reversible and irreversible injury patterns.

The 3.0-hr and 4.5-hr periods of ischemia resulted in distinct patterns of microvascular injury. After $3 \mathrm{hr}$ of ischemia there was some diffuse capillary obstruction but no arteriolar or venular obstruction. The major consequence of this injury was extravasation of the fluorosceinlabeled albumin which is manifested clinically as edema. Some capillary obstruction persisted. However the $4.5 \mathrm{hr}$ period of ischemia caused a much different pattern of injury. There were areas of primary "no reflow," that is, areas in which there was no return of circulation upon release of the clamps. This usually entailed loss of whole microvascular units consisting of the feeding arteriole, capillaries, and draining venules. In addition, there were many areas in which there was flow initially that eventually became obstructed during the later phases of the reperfusion period. These obstructions usually began within the capillaries. Thromboses of microvessels were rarely observed. In fact, in the capillaries without forward flow we observed columns of dye with multiple red blood cells which were either motionless or in an oscillating pattern. There was no major macrovessel thrombosis in any of the experimental preparations. This lack of extensive thrombosis either in the macro- or microcirculation occurred even though no heparin anticoagulation was used.

We believe that thrombosis of the microcirculation is not likely to be the primary cause of the loss of micro- 
vascular perfusion or the so-called no reflow phenomenon $[11,12]$. However, in areas in which there was no reflow after release of the clamps we are uncertain of the cause of the microvessel obstruction. In other studies using both light microscopy [13] and electron microscopy [12] neither platelet thrombi nor fibrin thrombi have been observed. In addition, a number of studies [12,14] have shown that heparinization fails to prevent the loss of microvascular perfusion that occurs after reperfusion of ischemic skeletal muscle or kidney.

There has been considerable interest in understanding the mechanisms that regulate neutrophil-endothelial cell interactions during inflammation and ischemia. The adhesion of neutrophils to the endothelium is thought to be an active process secondary to activation of both the neutrophil and the endothelial cell by common mediators such as complement which are found during inflammation or ischemia. A significant increase in the extent of white blood cell and endothelial cell interactions occurred for both the 3.0- and the $4.5 \mathrm{-hr}$ periods of ischemia. There was a significant increase in the 4.5 -hr ischemic group in the number of white cells sticking to the endothelium. This increased adhesion between the white blood cells and the microvascular endothelium did not occur at all levels of the microcirculation; it was seen exclusively within the postcapillary and draining venules. There was almost no permanent sticking of any white blood cells to arteriolar endothelium. There has been recent in vitro work showing that the aortic endothelial cells do not have the same receptors for white cell attachment as do venous endothelial cells [15]. This increased adhesion of white blood cells to the venular endothelium in the immediate postreperfusion period implies a role for the white cells beyond that of a late scavenger of necrotic cells. Rather, white blood cells probably have a early, central role in the amplification of the initial ischemic injury. Our model should permit further clarification of the mechanisms responsible for the increased adhesion of white blood cells to the microvascular endothelium.

An important question concerning the results of this study is how do these measures of microvascular injury relate to changes that may be observed at the level of the whole muscle. The pattern of changes in the $\mathrm{pH}$ of the muscle very closely paralleled the extent of microvascular injury that we observed (Fig. 4). Whereas the $\mathrm{pH}$ recovered to baseline levels after $3.0 \mathrm{hr}$ of ischemia, the $\mathrm{pH}$ remained below $7.0 \mathrm{pH}$ units at the same time period after $4.5 \mathrm{hr}$ of ischemia. The microvascular injury scoring system showed a greater degree of microvascular injury after $4.5 \mathrm{hr}$ of ischemia than after $3.0 \mathrm{hr}$. In addition, the 4.5$\mathrm{hr}$ ischemia group appeared to have an irreversible injury. In contrast, the 3.0 -hr group had good reflow but progressive extravasation. We believe that the latter represented a reversible microvascular injury pattern. Only with experiments examining later time periods after ischemia will we be able to conclusively show this. Finally, we believe our $\mathrm{pH}$ measurements to be accurate, because our baseline and subsequent measurements are similar to those obtained by other groups $[16,17]$.

There have been few other in vivo observations of the changes within the microcirculation after reperfusion of ischemic skeletal muscle. These other studies employed muscles such as the cremaster which are not true muscles of locomotion $[9,18]$. How relevant are changes after ischemia-reperfusion in muscles such as the cremaster to true muscles of locomotion such as the tibialis anterior is unknown. However, one study using transillumination of the cremaster muscle showed that $30 \%$ of the capillaries were obstructed after $30 \mathrm{~min}$ of ischemia and no further increase in capillary obstruction after $2 \mathrm{hr}$ of ischemia. This pattern of microvascular injury in the cremaster of the rat was different than that in the tibialis anterior of the hamster. In the cremaster, in addition to the lack any progression in the microvascular injury after longer durations of ischemia, there was no arteriolar or venular obstruction observed. Although the preparation and variables measured were different between these studies, fluorescent intravital microscopy using epiillumination has been shown to be a more precise technique to determine capillary density in vivo than transillumination techniques [19].

These results are the first in vivo observations of the pattern of microvascular injury and the extent of white blood cell adhesion in a true muscle of locomotion. This model should allow further clarification of the mechanisms responsible for the microvascular injury that occurs during reperfusion of ischemic skeletal muscle. Both the scoring system for the extent of microvascular injury and the index of white cell adhesion should permit quantitative analysis of the mechanisms by which various interventions may reduce the extent of injury after reperfusion of ischemic skeletal muscle.

\section{ACKNOWLEDGMENTS}

We gratefully acknowledge Dr. Geert Jan Tangelder for his generous gift of acridine red and the expert assistance of Ms. Cathy A. Pfaus in the preparation of this manuscript. Dr. Messina dedicates this work to Michael R. Golding, M.D., mentor and friend.

\section{REFERENCES}

1. Belkin, M., LaMorte, W. L., Wright, G., and Hobson, R. W. II. The role of leukocytes in the pathophysiology of skeletal muscle ischemic injury. J. Vasc. Surg. 10: 14, 1989.

2. Korthius, K. J., Ganger, D. N., Townsley, M. I., and Taylor, A. E. The role of oxygen-derived free radicals in ischemia-induced increases in canine skeletal muscle vascular permeability. Circ. Res. 57: 599, 1985.

3. Perry, M. O., and Fantini, G. Ischemia: Profile of an enemy:reperfusion injury of skeletal muscle. J. Vasc. Surg. 6: 231, 1987.

4. Sapega, A. A., Heppenstall, R. B., Park, Y. S., and Sokolow, D. P. Optimizing tourniquet application and release times in extremity surgery: A biochemical and ultrastructural study. J. Bone Joint Surg. 67: 303, 1985. 
5. Walker, P. M. V., Lindsay, T. F., Labbe, R., Mickle, D. A., and Romaschin, A. D. Salvage of skeletal muscle with free radical scavengers. J. Vasc. Surg. 5: 68, 1987.

6. Tangelder, G. J., Slaff, D. W., Muijtjens, A. M. M., Arts, T., oude Egbrink, M. G. A., and Reneman, R. S. Velocity profiles of blood platelets and red blood cells flowing in arterioles of the rabbit mesentery. Circ. Res. 59: 505, 1986.

7. House, S. D., and Lipowsky, H. H. Leukocyte-endothelium adhesion: microhemodynamics in mesentery of the cat. Microvasc. Res. 34: 363, 1987.

8. McDonagh, P. F., and Roberts, D. J. Prevention of transcoronary macromolecular leakage after ischemia-reperfusion by the calcium entry blocker nisoldipine: direct observations in isolated rat hearts. Circ. Res. 58: 127, 1986.

9. Suval, W. D., Duran, W. N., Boric, M. P., Hobson, R. W., II, Berendsen, P. B., and Ritter, A. B. Microvascular transport and endothelial cell alterations preceding skeletal muscle damage in isch emia and reperfusion injury. Amer. J. Surg. 154: 211, 1987.

10. Engler, R. L., Schmid-Schonbein, G. W., and Pavelec, R. S. Leukocyte capillary plugging in myocardial ischemia and reperfusion in the dog. Amer. J. Pathol. 11 1: 98, 1983.

11. Harman, J. W., and Gwinn, R. P. The recovery of skeletal muscle fibers from acute ischemia as determined by histologic and chemical methods. Amer. IJ Pathol 25: 741, 1949.
12. Strock, P. E., and Majno, G. Microvascular changes in acutely ischemic rat muscle. Surg. Gynecol. Obstet. 129: 1213, 1969.

13. Harman, J. W. The significance of local vascular phenomenon in the production of ischemic necrosis in skeletal muscle. Amer. $J$. Pathol. 24: 625, 1948.

14. Summers, W. K., and Jamison, R. L. The no-reflow phenomenon in renal ischemia. Lab. Invest. 25: 635, 1971.

15. Pohlman, T. H., Stanness, K. A., Beatty, P. G., Ochs, H. D., and Harlan, J. M. An endothelial cell surface factor(s) induced in vitro by lipopolysaccharide, interleukin 1 , and tumor necrosis factoralpha increases neutrophil adherence by a CD W 18-dependent mechanism. $J$. Immunol. 36(12): 4548, 1986.

16. Kost, G. J. Surface pH of the medial gastrocnemius and soleus muscles during hemorrhage shock and ischemia. Surgery 95: 183, 1984.

17. Hagberg, $\mathrm{H}$. Intracellular $\mathrm{pH}$ during ischemia in skeletal muscle: Relationship to membrane potential, extracellular pII, tissue lactic acid and ATP. Pflugers Arch. 404: 342, 1985.

18. Sack, F.-U., Funk, W., Hammersen, F., and Messmer, K. Microvascular injury of skeletal muscle and skin after different periods of pressure induced ischemia. Prog. Appl. Microcirc. 12: 282, 1987.

19. Damon, D. H., and Duling, B. R. Distribution of capillary blood flow in the microcirculation of the hamster: An in vivo study using epifluorescent microscopy. Microvasc. Res. 27: 81, 1984. 